\title{
Neuroimaging-Aided Prediction of the Effect of Methylphenidate in Children with Attention-Deficit Hyperactivity Disorder: A Randomized Controlled Trial
}

\author{
Ayaka Ishii-Takahashi' ${ }^{1,2,3}$, Ryu Takizawa ${ }^{3}$, Yukika Nishimura ${ }^{3}$, Yuki Kawakubo', Kasumi Hamada ${ }^{3,4}$, \\ Shiho Okuhata ${ }^{5,6}$, Shingo Kawasaki ${ }^{3,7}$, Hitoshi Kuwabara ${ }^{1,8}$, Takafumi Shimada ${ }^{3}$, Ayako Todokoro', \\ Takashi Igarashi $^{2,9}$, Kei-ichiro Watanabe ${ }^{10}$, Hidenori Yamasue ${ }^{3}$, Nobumasa Kato' ", Kiyoto Kasai*,3 and \\ Yukiko Kano' \\ 'Department of Child Neuropsychiatry, Graduate School of Medicine, The University of Tokyo, Tokyo, Japan; ${ }^{2}$ Department of Paediatrics, Graduate \\ School of Medicine, The University of Tokyo, Tokyo, Japan; ${ }^{3}$ Department of Neuropsychiatry, Graduate School of Medicine, The University of Tokyo, \\ Tokyo, Japan; ${ }^{4}$ Graduate School of Comprehensive Human Science, Graduate Course of Disability Sciences, University of Tsukuba, Tsukuba, Ibaraki, \\ Japan; ${ }^{5}$ Department of Electrical Engineering, Graduate School of Engineering, Kyoto University, Kyoto, Japan; ${ }^{6}$ Research Fellow of the Japan Society \\ for the Promotion of Science, Tokyo, Japan; ${ }^{7}$ Optical Topography Group, Application Development Office, Hitachi Medical Corporation, Chiba, \\ Japan; ${ }^{8}$ Disability Services Office, Communication Support Room, Division for Counseling and Support, the University of Tokyo, Tokyo, Japan; \\ ${ }^{9}$ National Centre for Child Health and Development, Tokyo, Japan; ${ }^{10}$ Division for Counseling and support, Graduate School of Medicine, \\ The University of Tokyo, Tokyo, Japan; "Karasuyama Hospital Showa University School of Medicine, Tokyo, Japan
}

Although methylphenidate hydrochloride (MPH) is a first-line treatment for children with attention-deficit hyperactivity disorder (ADHD), the non-response rate is 30\%. Our aim was to develop a supplementary neuroimaging biomarker for predicting the clinical effect of continuous MPH administration by using near-infrared spectroscopy (NIRS). After baseline assessment, we performed a double-blind, placebo-controlled, crossover trial with a single dose of MPH, followed by a prospective 4-to-8-week open trial with continuous MPH administration, and an ancillary I-year follow-up. Twenty-two drug-naïve and eight previously treated children with ADHD (NAIIVE and NON-NAIIVE) were compared with 20 healthy controls (HCs) who underwent multiple NIRS measurements without intervention. We tested whether NIRS signals at the baseline assessment or $\triangle$ NIRS (single dose of MPH minus baseline assessment) predict the Clinical Global Impressions-Severity (CGI-S) score after 4-to-8-week or I-year MPH administration. The secondary outcomes were the effect of MPH on NIRS signals after single-dose, 4-to-8-week, and I-year administration. $\triangle$ NIRS significantly predicted CGI-S after 4-to-8-week $\mathrm{MPH}$ administration. The leave-one-out classification algorithm had $81 \%$ accuracy using the NIRS signal. $\Delta \mathrm{NIRS}$ also significantly predicted CGI-S scores after I year of MPH administration. For secondary analyses, NAÏVE exhibited significantly lower prefrontal activation than HCs at the baseline assessment, whereas NON-NAIIVE and HCs showed similar activation. A single dose of MPH significantly increased activation compared with the placebo in NAïVE. After 4-to-8-week administration, and even after MPH washout following I-year administration, NAIIVE demonstrated normalized prefrontal activation. Supplementary NIRS measurements may serve as an objective biomarker for clinical decisions and monitoring concerning continuous MPH treatment in children with ADHD.

Neuropsychopharmacology (2015) 40, 2676-2685; doi: 10.1 038/npp.20I5.I28; published online 27 May 2015

\section{INTRODUCTION}

Individualized medicine (Erder et al, 2012) using biomarkers such as neuroimaging ('third-generation' or 'next-generation' neuroimaging) is increasingly important in psychiatry (Borgwardt and Fusar-Poli, 2012). The psychostimulant methylphenidate hydrochloride (MPH) is an effective firstline treatment for children with attention-deficit hyperactivity

* Correspondence: Professor K Kasai, Department of Neuropsychiatry, Graduate School of Medicine, The University of Tokyo, 7-3-1 Hongo, Bunkyo-ku, Tokyo II3-8655, Japan, Tel: +8I 35800 89|9, Fax: +8I 3 5800 9|62, E-mail: kasaik-tky@umin.net

Received 20 November 2014; revised 28 February 20I5; accepted 27 March 2015; accepted article preview online 4 May 2015 disorder (ADHD) (Buitelaar and Medori, 2010). However, $\sim 30 \%$ of children with ADHD do not respond to $\mathrm{MPH}$ (Wilens et al, 2008) and experience no benefit or only adverse side effects (Cho et al, 2007; Rapport et al, 1994). Moreover, although positron emission tomography studies of MPH exposure suggest that catecholamine dysfunction at least partially mediates the behavioral and cognitive features of ADHD (Hannestad et al, 2010; Krause, 2008; Volkow et al, 2002), how MPH affects the nervous system remains unclear, especially over long periods.

Several studies have attempted to predict MPH efficacy using clinical characteristics including age (Buitelaar et al, 1995; Zeiner et al, 1999), IQ (Buitelaar et al, 1995), symptoms, disorder severity (Buitelaar et al, 1995; Zeiner et al, 1999), and 
Table I Demographics of the NAÏVE, NON-NAÏVE, and Healthy Control Subjects at Baseline Assessment

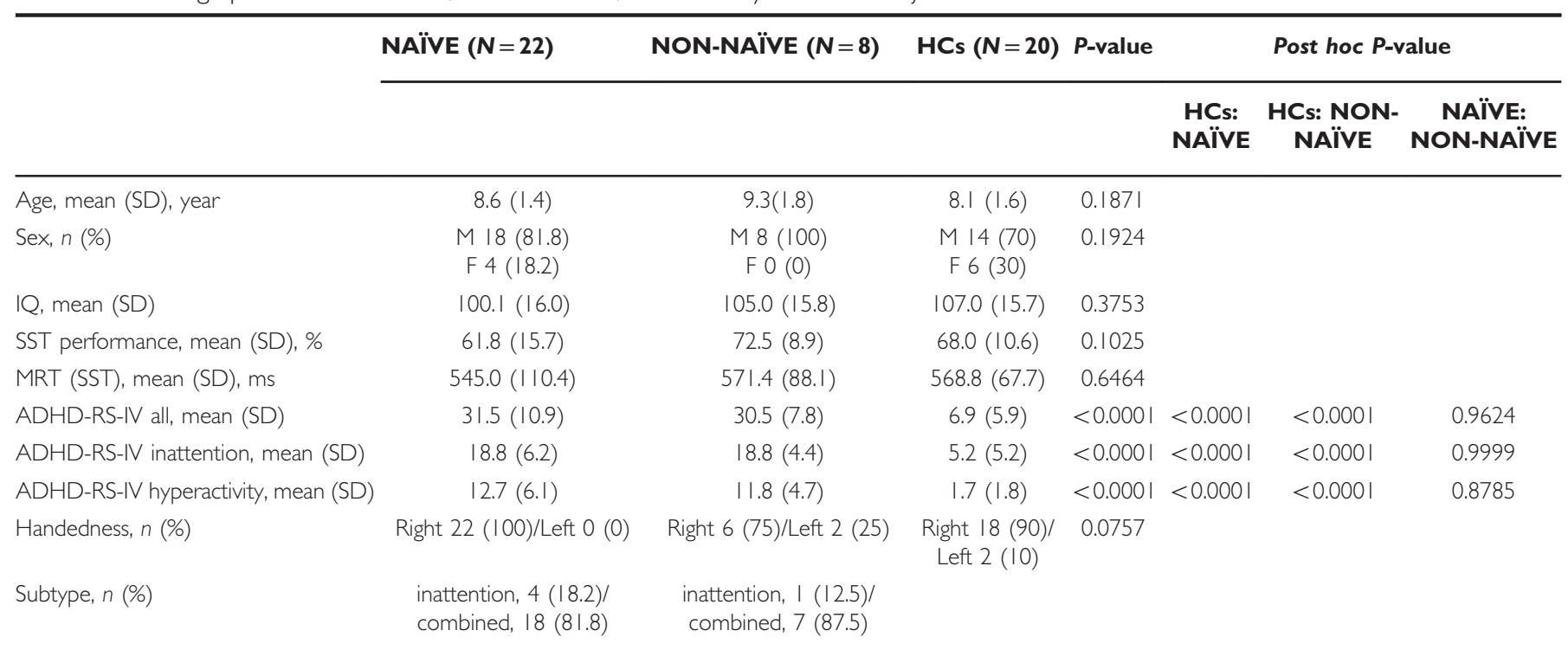

Abbreviations: ADHD-RS-IV, attention-deficit hyperactivity disorder rating scale IV; HCs, healthy controls; IQ, intelligence quotient; MRT, mean reaction time; NAÏVE, ADHD-drug naïve; NON-NAÏVE, ADHD patients who had taken methylphenidate for several months; SST, stop signal task.

neuropsychological test scores (Lee et al, 2009). Recently, more objective biomedical predictors (Hermens et al, 2006) of brain function (An et al, 2013; Cho et al, 2007; Ilgin et al, 2001; la Fougere et al, 2006; Sangal and Sangal, 2004; Schweitzer et al, 2003) have been used (see also Supplementary Materials). Most neuroimaging MPH trials have included previously medicated subjects. However, recent studies have revealed differences in brain structure between drug-naïve and previously treated ADHD patients (Frodl and Skokauskas, 2012; Shaw et al, 2009).

Here, we primarily aimed to develop an objective marker using near-infrared spectroscopy (NIRS) that predicts the efficacy of continuous MPH administration for children with ADHD. NIRS imaging is safe, portable, and allows easy examination of subjects in a natural sitting position (IshiiTakahashi et al, 2013; Monden et al, 2012; Takizawa et al, 2008). To elicit prefrontal activation, we used the stop signal task (SST), an inhibitory task that reflects the pathophysiology of ADHD (Hart et al, 2013; Rubia et al, 2014). The single-dose $\mathrm{MPH}$ administration phase used a randomized, double-blind, placebo-controlled, crossover design, and the subsequent outcome was assessed in a 4-to-8-week open trial and 1-year follow-up. To our knowledge, no previous study has followed patients for 1 year to predict the effect of $\mathrm{MPH}$ using neuroimaging. We hypothesized that prefrontal activation at baseline assessment, or the difference in NIRS signal between a single administration of $\mathrm{MPH}$ and the baseline assessment, would predict clinical improvement after mid-term (4-to-8-week) and long-term (1-year) $\mathrm{MPH}$ treatment in drug-naïve patients with ADHD. Notably, we divided children with ADHD into those who had not received any medication (drug naïve) and those who had received chronic treatment with $\mathrm{MPH}$ (non-naïve) because the NIRS signals at baseline may be altered by previous $\mathrm{MPH}$ treatment.

Our secondary purpose was to test whether multiple NIRS measurements are useful in monitoring prefrontal activation during continuous MPH treatment in children with ADHD. For this purpose, we recruited healthy controls (HCs) and monitored their NIRS signals at multiple times without any intervention. Then, we tested the effect of MPH treatment on the NIRS signals after single-dose, mid-term, and long-term administration in ADHD children.

\section{MATERIALS AND METHODS}

\section{Participants}

We examined 30 children with ADHD (mean age: $8.6 \pm 1.4$ years) and 20 age-, sex-, and IQ-matched HCs (Table 1), all recruited from an outpatient unit at the University of Tokyo Hospital, who consisted of two groups: drug-naïve ADHD (NAÏVE) and ADHD patients taking MPH for at least 1 month (NON-NAÏVE; Figures 1 and 2). HCs underwent multiple neuroimaging measurements without any clinical intervention.

Child psychiatrists diagnosed ADHD through comprehensive clinical assessments and in accordance with the DSMIV. Current and lifetime DSM-IV diagnoses were determined by a consensus decision based on the results of independent clinical interviews, clinical data, and the Mini-International Neuropsychiatric Interview for Children and Adolescents (Otsubo et al, 2005). Additional inclusion criteria were age (6-12 years) and IQ (above 70) determined with the Wechsler Children Intelligence Scale, 3rd edition. The exclusion criteria are described in Supplementary Materials.

The ADHD Rating Scale IV (ADHD-RS-IV) was used to assess ADHD symptoms (DuPaul et al, 1998). Problematic behaviors were measured from parent reports using the Child Behavior Checklist (CBCL; Achenbach et al, 1991).

\section{Trial Design}

After baseline assessment before MPH administration (Phase 1), we conducted a double-blind, placebo-controlled, 


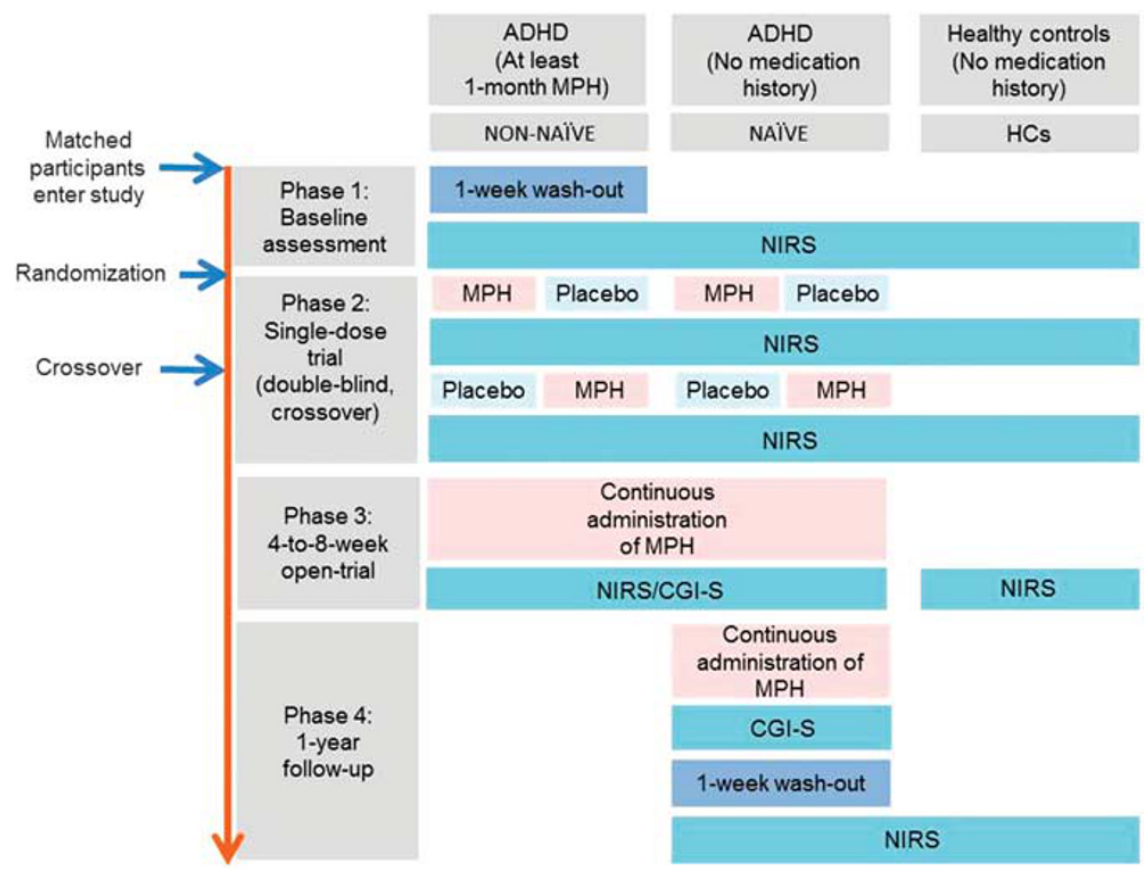

Figure I Schematic diagram of the trial protocol. NAÏVE and NON-NAÏVE underwent baseline assessment (Phase I), a single-dose trial (Phase 2), and a 4-to-8-week open trial (Phase 3). NAÏVE participants also underwent a I-year follow-up (Phase 4). NAÏVE was evaluated by NIRS in five sessions (Phase I: I session, Phase 2: 2 sessions, Phase 3: I session, and Phase 4: I session). NON-NAÏVE was evaluated by NIRS signal in four sessions (Phase I: I session, Phase 2: 2 sessions, and Phase 3: I session). We evaluated the NIRS signal of HCs, who did not take MPH, at the same intervals and times as NÄ̈VE to detect the effects of the repeated measurement. ADHD, attention-deficit hyperactivity disorder; HCs, healthy controls; MPH, methylphenidate hydrochloride; NAïVE, ADHD-drug NAÏVE; NIRS, near-infrared spectroscopy; NON-NAÏVE, ADHD patients who had taken methylphenidate for several months.

crossover, prospective trial of single-dose MPH administration (Phase 2), with a subsequent 4-to-8-week open trial (Phase 3; Figure 1). We then conducted a 1-year follow-up (Phase 4) as an ancillary study. Prefrontal hemodynamics were measured in NAÏVE and NON-NAÏVE using NIRS at Phase 1, Phase 2 (two times after a single dose of MPH or placebo), and Phase 3. NAÏVE was also assessed at Phase 4. We evaluated the NIRS signals of HCs at the same time points used for NAÏVE (Figure 1). For details about the repeated measurements in HCs, see Supplementary Materials.

The target sample size for this study was $\sim 20$ children (NAÏVE) for the primary outcome, after accounting for dropout cases. We estimated the sample size based on an optimal design for functional brain imaging (Carter et al, 2008).

All children and parents provided written informed consent after receiving a complete explanation of the study, according to the Declaration of Helsinki. The institutional review board (IRB) and ethical committee of the University of Tokyo Hospital approved this study (approval number: P2007009 and 630-8). This trial is registered as number UMIN000001270.

\section{Clinical Interventions}

A schematic diagram of the trial protocol is shown in Figure 1. In Phase 1, NON-NAÏVE was instructed to stop taking MPH for 1 week before the single-dose trial began.

In Phase 2, placebo or MPH (18 mg; MPH osmotic-release oral system (OROS), Concerta) was administered at 1-week intervals. Subjects received a blinded capsule (placebo or $\mathrm{MPH}$ ) in the front of the investigator (AI-T) $5 \mathrm{~h}$ before the NIRS session.

In Phase 3, the optimal dose was administered for 4 weeks. After a 1-week washout period, the NON-NAÏVE group was administered the same amount of MPH that they were taking before participating in the trial. Because the NON-NAÏVE group did not experience a titration period, the length of their open-label trial was 4 weeks. In the NAÏVE group, the dose was determined from the results of the controlled medication trial described below. The ADHD-RS-IV and side-effect rating scale were assessed weekly. A psychiatrist reviewed the data each week to determine the best MPH dose. MPH was titrated to an optimal response from an initial dose of $18 \mathrm{mg}$. During titration, the dosage was increased by $9 \mathrm{mg}$ at weekly visits. When side effects occurred, the dose was reduced to a level where no side effects occurred, which then was considered the optimal dose. After the titration period, the NAIIVE group was administered the optimal dose for 4 weeks. After titration, the optimal dose for each patient was determined (mean dose: $25.4 \mathrm{mg}, 0.91 \mathrm{mg} / \mathrm{kg}$; SD: 5.9 ; median: 27). Participants continued receiving the optimal dose of MPH for 4 weeks (mean duration including titration: 5.45 weeks; SD: 1.29 ; median: 5).

Phase 4 was a 1-year follow-up as an ancillary study. We followed NAÏVE participants who continued MPH administration for at least 1-year (mean duration: 14.7 months; SD: 2.3; median: 14.5) after the 4-to-8-week open trial. The same child psychiatrist who evaluated the children in the baseline assessment evaluated the severity and clinical response after 1-year-MPH administration using the Clinical Global 


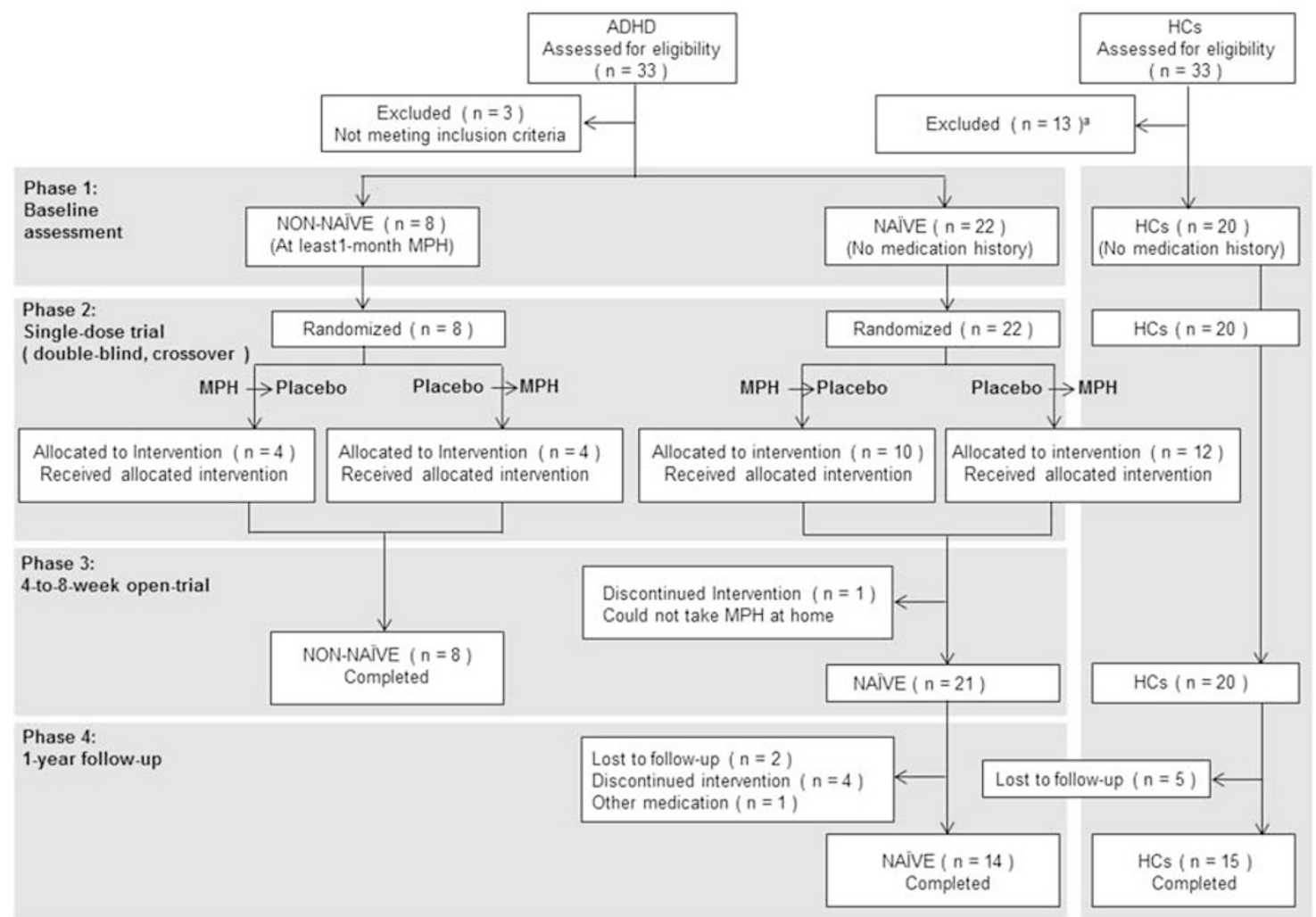

Figure 2 Trial profile. For the primary outcome, only NAÏVE $(N=21)$ was assessed. For the secondary outcome, NAÏVE (N=22) and NON-NAïVE $(N=8)$ groups were evaluated for the effect of the single-dose trial. NAïVE $(N=21)$, NON-NAïVE $(N=8)$, and $H C s(N=20)$ were evaluated in the analysis for the effect of the 4-to-8-week open trial. For the ancillary analysis, naïve was analyzed to determine the predictor of CGI-S after I year of MPH administration $(N=14)$. NAÏVE $(N=14)$ and HCs $(N=15)$ were analyzed for the effect of I year of MPH administration after washout. (a) After participants were matched for age, sex, IQ, and SST performance among the NAÏVE, NON-NAÏVE, and HCs, 20 members of HCs were selected. ADHD, attention-deficit hyperactivity disorder; HCs, healthy controls; MPH, methylphenidate hydrochloride; NAÏVE, ADHD-drug naïve; NIRS, near-infrared spectroscopy; NONNAÏVE, ADHD patients who had taken methylphenidate for several months; SST, stop signal task.

Impressions-Severity (CGI-S). The NAÏVE stopped taking MPH for 1 week before undergoing the last NIRS measurement.

The washout period, timing of $\mathrm{MPH}$ administration, titration procedure, and mean dose of $\mathrm{MPH}$ are described in the Supplementary Materials.

\section{Outcomes}

We used NIRS to measure changes in the mean oxygenated hemoglobin concentration ([oxy-Hb]) in the bilateral inferior frontal cortex (IFC: Supplementary Figure S1) during the SST, which served as an explanatory variable. The procedures for the SST and NIRS measurements are described in detail in Supplementary Materials and Supplementary Figure S2, and in our previous report (Ishii-Takahashi et al, 2013).

We also used the CGI-S (Guy, 1976) scores after 4-to-8week administration (Phase 3) and 1-year administration (Phase 4) of the optimal dose of MPH as outcome measures (Figure 1). We did not use the ADHD-RS-IV as a primary outcome measure in the clinical trial registry because ADHDRS-IV was translated to Japanese in 2007 and has not been validated in Japanese patients. A detailed explanation about the adoption of CGI-S as an assessment tool of primary outcome was reported in Supplementary Materials. The child psychiatrist (AI-T) reviewed the severity and clinical response as assessed by CGI-S (Buitelaar et al, 1995; Cho et al, 2007).
For the primary analysis, regression analysis was conducted to examine whether [oxy-Hb] measured at the baseline assessment or $\Delta[$ oxy-Hb] (single dose of $\mathrm{MPH}$ minus baseline assessment) predicted CGI-S scores after the 4-to8 -week open trial and after 1 year of MPH administration.

For secondary analysis, we examined at Phase 1, whether brain function differed among NAÏVE, NON-NAÏVE, and HCs; at Phase 2, whether a single dose of MPH or placebo-affected [oxy-Hb]; at Phase 3, whether brain function differed among NAÏVE, NON-NAÏVE, and HCs during the 4-to-8-week administration of $\mathrm{MPH}$; and at Phase 4, whether administration of MPH for 1-year affected [oxy-Hb] after washout.

\section{Randomization and Blinding of Phase 2}

After baseline assessments, a study investigator (AI-T) assigned the participants to an intervention. An IRB member (Y.A.) generated a random allocation sequence to blind participants assigned an intervention and stratified the participants into two groups according to their medications. The patients were randomly assigned in a 1:1 ratio to one of the two groups. In the first group, the patients first received a single dose of $\mathrm{MPH}$, and 1 week later, they received a single dose of placebo. In the second group, the order was reversed. Participants, care providers, and investigators were doubleblinded to the intervention. Placebos were sucrose capsules 
similar to the MPH capsules. The investigators were informed of patient assignments after Phase 3 was completed.

\section{Statistical Analyses}

Statistical analyses were conducted after completing the trials and opening the allocation; the analyses followed the intention-to-treat principle. All reported $P$-values are twotailed. Clinical and behavioral results were considered significant at $P<0.05$. The $95 \%$ confidence interval $(95 \%$ $\mathrm{CI})$ and effect size $(d)$ are shown when applicable. Analyses were conducted with IBM SPSS software (version 20.0). At the baseline assessment, between-group differences in clinical scores and SST performance were tested using a one-way analysis of variance (ANOVA) with Tukey's Honestly Significant Difference (HSD) post hoc test. The $\chi^{2}$-test was used to determine differences in sex and handedness. Mean reaction time during the SST, SST performance, and ADHDRS-IV were analyzed using a two-way repeated-measures ANOVA with post hoc Tukey's HSD test.

The primary analyses were conducted for NAÏVE. A stepwise multiple regression analysis was conducted with CGI-S scores obtained after the 4-to-8-week open trial as a dependent variable with the [oxy-Hb] at the baseline assessment in the right IFC (RIFC) and left IFC (LIFC), $\Delta$ [oxy-Hb] (single dose of MPH minus baseline assessment) in the RIFC and LIFC, and 11 demographic and clinical variables as independent variables: MPH dosage, ADHD-RSIV scores ('Inattention' and 'Impulsivity and Hyperactivity' sub-scores and total score), CBCL scores ('Internalizing' and 'Externalizing' sub-scores and total score), SST task performance, IQ, age, and sex. We used $t$-tests to identify differences in $\Delta[\mathrm{oxy}-\mathrm{Hb}$ ] (single dose of MPH minus baseline assessment) between subjects with a CGI-S score $\leqslant 3$ and $\geqslant 4$. We then submitted the resulting values to a parametric Fisher linear discriminant analysis classification algorithm (Ponseti et al, 2012) to discriminate CGI-S $\leqslant 3$ from CGI-S $\geqslant 4$. Additional information about the definition of responder and the cross-validation methods are presented in the Supplementary Materials. We also performed a stepwise multiple regression analysis after administration of MPH for 1 year with CGI-S scores as the dependent variable, and mean $[\mathrm{oxy}-\mathrm{Hb}]$ change at the baseline assessment, $\Delta$ [oxy-Hb] (single dose of MPH minus baseline assessment), and the 11 demographic and clinical variables described above as independent variables.

For the secondary analyses, to detect differences among NAÏVE, NON-NAÏVE, and HCs at the baseline assessment (Phase 1) and after the 4-to-8-week open-trial (Phase 3), the [oxy-Hb] was subjected to a repeated-measures ANOVA with group (NAÏVE, NON-NAÏVE, and HCs) as a betweensubject factor and NIRS session (Phase 1 and 3 ) and laterality (RIFC and LIFC) as within-subject factors. At Phase 2, we identified differences in NIRS signals between MPH and placebo administration in the single-dose trial; the [oxy- $\mathrm{Hb}$ ] was subjected to a repeated-measures ANOVA with group (NAÏVE and NON-NAÏVE) as a between-subject factor and NIRS session (single dose of MPH and placebo) and laterality (RIFC and LIFC) as within-subject factors. To identify differences in NIRS between NAÏVE and HCs at the baseline assessment and after washout following MPH administration for 1 year, [oxy-Hb] was subjected to a repeated-measures
ANOVA with group (NAÏVE and HCs) as a between-subject factor and NIRS session (Phase 1 and 4) and laterality (RIFC and LIFC) as within-subject factors. Subsequently, we used the Bonferroni correction for specific post hoc contrasts (significance at Bonferroni-corrected $P<0.05$ ).

\section{RESULTS}

\section{Participants}

The study began on 1 August 2008 and ended with the 1-year follow-up termination on 29 June 2011. Table 1 presents the baseline demographic characteristics for the study participants. Of the 33 potential participants with ADHD, 3 were excluded for not meeting the inclusion criteria (Figure 2). Thus, we examined 30 patients in Phase 2 (22 NAÏVE and 8 NON-NAÏVE). One NAÏVE participant was excluded because of an inability to continue taking MPH during Phase 3. Among 21 NAÏVE patients who participated in Phase 3, 14 completed Phase 4, 4 stopped taking MPH, 2 stopped attending the hospital, and 1 changed medications. In addition, $20 \mathrm{HCs}$ were included in the analysis after assessment of 33 children for eligibility; 15 completed the follow-up, and 5 stopped attending the study. NAÏVE, NONNAÏVE, and HCs did not differ in SST performance (Table 1). The ADHD-RS-IV scores of both NAÏVE and NON-NAÏVE were significantly higher compared with those of HCs. Changes in performance and severity (baseline assessment and 4-to-8-week open trial), the comparison of demographic characteristics at the baseline assessment between NAÏVE and HCs participated in 1-year follow-up, and changes in performance and severity (baseline assessment and 1-year follow-up) in NAÏVE are described in Supplementary Materials, and Supplementary Table S1-S3.

\section{Outcomes}

\section{Primary results}

Predictor of CGI-S after 4-to-8-week open trial. In the 4-to-8-week open trial, there were 4 participants in CGI-S 2, 12 in CGI-S 3, and 5 in CGI-S 4. Multiple regression analysis for CGI-S scores after the 4-to-8-week open trial revealed a significant regression only for $\Delta$ [oxy-Hb] in LIFC $(R=0.519$, $P=0.0160, \quad \beta=-0.519, \quad 95 \% \quad \mathrm{CI}=-4.680 \quad$ to -5.440 ; Figure 3a). The $\Delta[$ oxy-Hb] in LIFC was significantly lower in participants with CGI-S $\geqslant 4$ than in those with CGI-S $\leqslant 3$ $(P=0.0084, d=1.200,95 \% \mathrm{CI}=0.351$ to 2.541$)$. The leaveone-out classification algorithm had an $81 \%(95 \% \mathrm{CI}=0.782$ to 0.813 ) accuracy $(81.3 \%$ sensitivity $(95 \% \mathrm{CI}=0.782$ to 0.820 ) and $80 \%$ specificity ( $95 \% \mathrm{CI}=0.779$ to 0.797$)$ ) using the $\Delta[\mathrm{oxy}-\mathrm{Hb}]$ in LIFC.

Predictor of CGI-S after 1-year of MPH administration. At the 1-year follow-up, there were six participants in CGI-S 2, six in CGI-S 3, and two in CGI-S 4. Multiple regression analysis for the CGI-S score at the 1-year follow-up revealed that $\Delta$ [oxy$\mathrm{Hb}$ ] in LIFC also predicted the CGI-S score $(R=0.716$, $P=0.0040, \beta=-0.716,95 \% \mathrm{CI}=-6.527$ to -1.562 ; Figure $3 \mathrm{~b}$ ).

\section{Secondary results}

Group comparison at baseline assessment and effect of 4-to-8-week MPH administration (Phase 1 and 3). We 
a

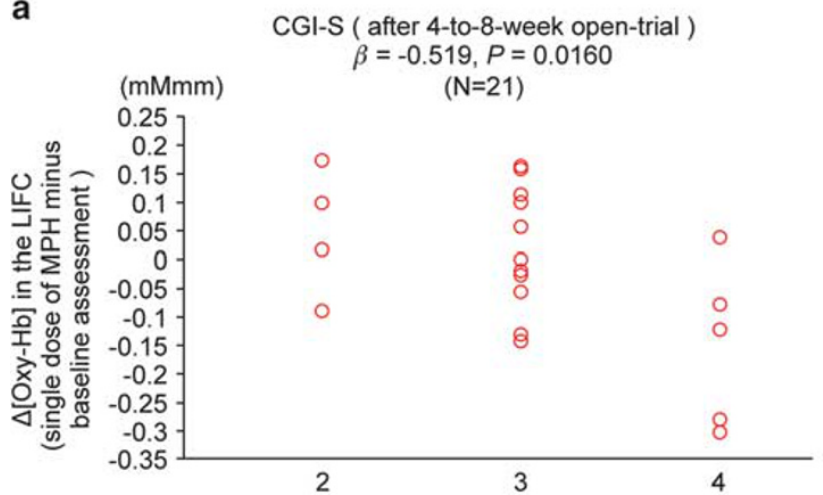

b

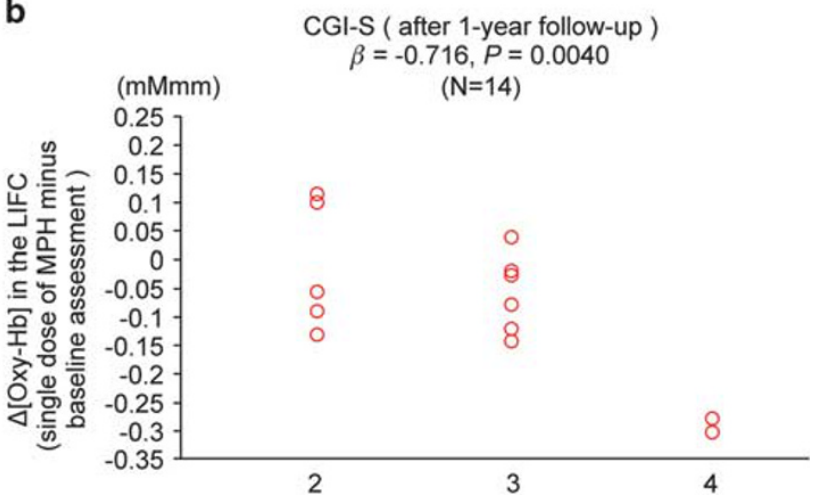

Figure 3 Results for primary outcomes: predictor of CGI-S in NAÏVE. (a) Correlation between CGI-S scores (4-to-8-week open trial: 2, borderline mentally ill $(N=4)$; 3 , mildly ill $(N=12) ; 4$, moderately ill $(N=5)$ ) and $\Delta[\mathrm{oxy}-\mathrm{Hb}]$ (single dose of MPH minus baseline assessment) in the LIFC $(\beta=-0.5|9, P=0.0| 60)$. (b) Correlation between CGI-S (after I-year follow-up: 2, borderline mentally ill $(N=6) ; 3$, mildly ill $(N=6) ; 4$, moderately ill $(N=2))$ and $\Delta[0 x y-H b]$ (single dose of MPH minus baseline assessment) in the LIFC $(\beta=-0.716, P=0.0040)$. ADHD, attention-deficit hyperactivity disorder; CGI-S, Clinical Global Impression-Severity; HCs, healthy controls; LIFC, left inferior frontal cortex; MPH, methylphenidate hydrochloride; NAÏVE, ADHD-drug naïve; NIRS, nearinfrared spectroscopy; NON-NAïVE, ADHD patients who had taken methylphenidate for several months; [oxy-Hb], oxygenated hemoglobin concentration; RIFC, right inferior frontal cortex.

observed a significant interaction for group (NAÏVE $v s$ NON-NAÏVE $v s$ HC) $\times$ NIRS session (baseline assessment $v s$ 4-to-8-week open trial) $\times$ laterality (RIFC $v s$ LIFC; $\mathrm{F}=3.743$, $P=0.0312)$. At the baseline assessment, NAÏVE demonstrated a significantly lower activation in the RIFC (Bonferroni-corrected $P=0.0455, d=-0.778,95 \% \mathrm{CI}=-1.452$ to $-0.178)$ and a tendency toward lower activation in the LIFC (corrected $P=0.0908, d=-0.729,95 \% \quad \mathrm{CI}=-1.424$ to - 0.153) compared with HCs (Figure 4a). However, NONNAÏVE and HCs did not differ significantly (Figure 4a). After the 4-to-8-week open trial, [oxy-Hb] did not differ significantly among NAÏVE, NON-NAÏVE, and HCs (Figure 4c). Additionally, HCs showed significantly lower bilateral IFC activation in the 4-to-8-week open trial than in the baseline assessment (RIFC; Bonferroni-corrected $P=0.0017, d=0.888$, 95\% CI $=0.204-1.499$ and LIFC; Bonferroni-corrected $P=0.0011, d=1.170,95 \% \mathrm{CI}=0.256$ to 1.557$)$. The details about the interaction of laterality and session in each group are described in the Supplementary Materials.

Effect of single-dose MPH administration (Phase 2). In the single-dose trial, a significant interaction was observed for group (NAÏVE $v s$ NON-NAÏVE) $\times$ NIRS session $(\mathrm{MPH}$ $v s$ placebo) $\times$ laterality (RIFC $v s$ LIFC; $\mathrm{F}=5.058, P=0.0326)$. NAÏVE demonstrated significantly higher activation in only the RIFC during the SST with MPH compared with the placebo (Bonferroni-corrected $P=0.0281, d=0.579,95 \%$ $\mathrm{CI}=0.009$ to 1.218 ; Figure $4 \mathrm{~b}$ ). In NON-NAÏVE, the effects of $\mathrm{MPH}$ and placebo did not differ significantly in the bilateral IFC.

Effect of 1-year MPH administration after washout (Phase 4). After the 1-year follow-up, a significant interaction was observed for group (NAÏVE vs HCs) $\times$ NIRS session (baseline assessment $v s$-year follow-up; $\mathrm{F}=8.160$, $P=0.0081)$. NAÏVE showed significantly lower activation in the bilateral IFC compared with the HCs group (Bonferronicorrected $P=0.0121, d=-0.938,95 \% \quad \mathrm{CI}=-1.741$ to -0.201 ; Supplementary Figure S3) at the baseline assessment.
No significant difference was observed between NAÏVE and HCs after washout of 1-year administration (Figure 4d). HCs demonstrated significantly lower bilateral IFC activation at the 1-year follow-up than at the baseline assessment (Bonferroni-corrected $P=0.0102, d=0.719, \quad 95 \% \quad \mathrm{CI}=$ -0.017 to 1.460$)$.

\section{Harm}

Side effects included loss of appetite $(N=18)$, difficulty falling asleep $(N=2)$, and transient tics $(N=2)$. These side effects diminished during titration. No serious side effects occurred.

\section{DISCUSSION}

This clinical trial is the first to demonstrate that a difference in prefrontal hemodynamic responses after single administration of MPH relative to the baseline level significantly predicted mid-term (4-to-8-week) and long-term (1-year) clinical efficacy of MPH in children with ADHD (Figure 3); the leave-one-out classification algorithm produced $81 \%$ accuracy. Additionally, (1) at the baseline assessment, NAÏVE exhibited significantly lower prefrontal activation than HCs, whereas NON-NAİVE and HCs showed similar activation; (2) a single dose of MPH significantly increased activation compared with the placebo in NAÏVE; and (3) after the 4-to-8-week administration, and even after MPH washout following 1-year administration, naïve demonstrated normalized prefrontal activation (Figure 4). Collectively, these results indicate that supplementary NIRS measurements may serve as a safe and simple objective biomarker for clinical decisions and monitoring for continuous MPH treatment for children with ADHD. The simplicity of NIRS measurement is also an important advantage for its clinical application in child psychiatry.

The strengths of our study include its randomized, doubleblind, placebo-controlled, crossover design; 1-year follow-up prospective study; and comparison of drug-naïve and $\mathrm{MPH}-$ treated children. The follow-up rate for the primary outcome was high $(96.7 \%)$. Therefore, the risk of biased conclusions 


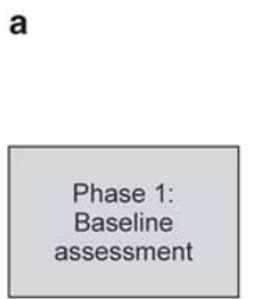

b

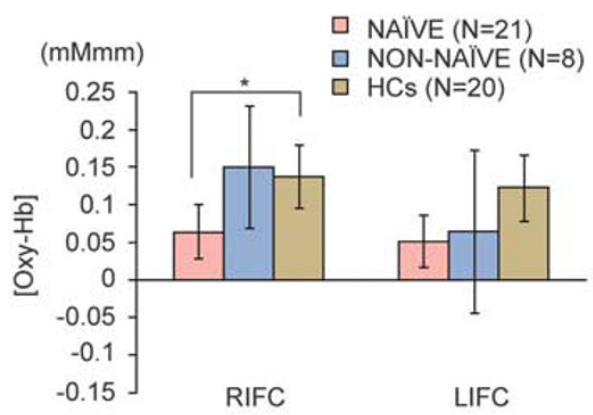

Phase 2:

Single-dose trial (double-blind, crossover)

C
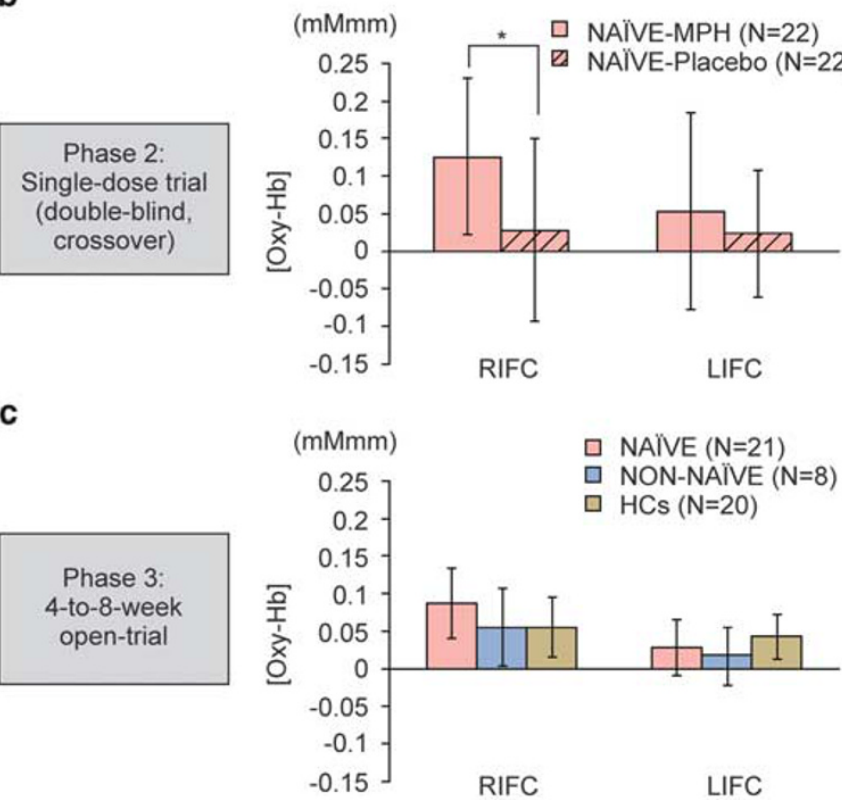

d
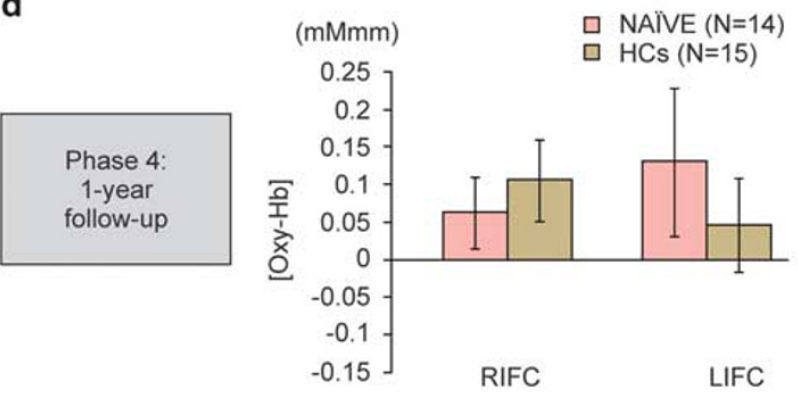

Figure 4 Results for secondary outcomes: effects of the 4-to-8-week open trial of MPH, effects of a single-dose trial, and effects of I year of MPH administration observed after washout. (a) At the baseline assessment (RIFC, Bonferroni-corrected $P=0.0455$; LIFC, Bonferroni-corrected $P=0.0908$ ). (b) Differences in $[\mathrm{oxy}-\mathrm{Hb}]$ changes during the SST in the single-dose trial between MPH and placebo treatments in NAIIVE (RIFC: Bonferronicorrected $P=0.028 \mathrm{I}$ ). (c) Differences in $[\mathrm{oxy}-\mathrm{Hb}$ ] changes during the SST between the HCs and ADHD groups after the 4-to-8-week open trial. Error bars indicate $95 \% \mathrm{Cl}$. (d) Differences in $[\mathrm{oxy}-\mathrm{Hb}$ ] changes during SST between the HCs and NAÏVE after I-year follow-up (baseline assessment: Bonferroni-corrected $P=0.0121$; after I-year follow-up: Bonferronicorrected $P=0.6386$ ). Error bars indicate $95 \% \mathrm{Cl}$. ADHD, attentiondeficit hyperactivity disorder; CGI-S, Clinical Global Impression-Severity; $\mathrm{HCs}$, healthy controls; LIFC, left inferior frontal cortex; MPH, methylphenidate hydrochloride; NAÏVE, ADHD-drug naïve; NIRS, near-infrared spectroscopy; NON-NAÏVE, ADHD patients who had taken methylphenidate for several months; [oxy-Hb], oxygenated hemoglobin concentration; RIFC, right inferior frontal cortex; SST, stop signal task. was low. The timing of NIRS ( $5 \mathrm{~h}$ after taking MPH) was selected to achieve the peak serum level of MPH OROS.

The main finding of our study is that changes in prefrontal activation after a single dose of MPH predicted the efficacy of long-term MPH administration (Figure 3). Increases in LIFC activity were previously observed in patients with ADHD who performed an error-monitoring task after acute (Rubia et al, 2011) and 6 weeks-MPH treatment during interference inhibition (Bush et al, 2008). A change in activation in the single-dose trial toward the level in HCs indicated a better response to continuous administration of MPH. These results are in line with a magnetic resonance imaging (MRI) study that predicted the response to continuous MPH administration using resting-state signals (An et al, 2013). We observed no correlation between MPH efficacy and baseline characteristics such as ADHD-RS-IV, CBCL internalizing score, age, IQ, or sex, which is consistent with the outcomes reported for a previous event-related potential study (Hermens et al, 2005). Although other neuroimaging modalities have predicted the effects of MPH (An et al, 2013; Cho et al, 2007; Ilgin et al, 2001; la Fougere et al, 2006; Sangal and Sangal, 2004; Schweitzer et al, 2003), we used NIRS because of its simplicity and safety, which are beneficial in a clinical setting. Because only NAÏVE were used to predict the effect of MPH, supplementary NIRS measurements may be applied in clinical situations in which a physician must decide whether a child should begin taking the medication.

Although it was not a primary focus of our study, we confirmed previous findings, obtained using other neuroimaging modalities, of prefrontal abnormalities in ADHD and their normalization by MPH treatment. First, NAÏVE demonstrated lower activation in the RIFC at the baseline assessment (Figure 4a), consistent with previous findings in drug-naïve children with ADHD (Hart et al, 2013). In contrast, NON-NAÏVE demonstrated activation similar to that of $\mathrm{HCs}$ at the baseline assessment, thus indirectly indicating the effect of previously administered $\mathrm{MPH}$, which is in line with the findings of a previous functional MRI (fMRI) study in adults (Schlochtermeier et al, 2011; Stoy et al, 2011). Second, a single dose of MPH increased the right frontal NIRS signal (Figure $4 \mathrm{~b}$ ), thus confirming that MPH increased RIFC activation during an inhibitory control task (Rubia et al, 2014). Third, the change toward a level of prefrontal activation similar to that for HCs after the 4-to-8week open trial (Figure 4c) is consistent with the results from a fMRI study of adults with ADHD (Bush et al, 2008) and an ERP study in children with ADHD (Sawada et al, 2010). Finally, our finding (Figure 4d) that long-term (1 year) MPH treatment affected brain development in ADHD children is consistent with the findings of a prospective MRI study (Konrad et al, 2007).

In the current study, no significant differences in SST performance were observed between children with ADHD and HCs. To detect the differences between ADHD and control groups using only SST as in previous studies (de Vries and Geurts, 2014; Gupta and Kar, 2009), the task would need to contain more trials than those included in our task. We designed our cognitive task such that the performance of the different groups did not significantly differ because we intended to detect significant differences in the NIRS signal during a short cognitive task. Additionally, the degree of 
difficulty of our SST varied according to the ability of the participants as described in the supplementary section, which likely also contributed to the lack of significant difference in SST performance between the ADHD and HCs groups.

Some limitations of our study should also be highlighted. First, methodological limitations include the smaller number of participants for NON-NAÏVE $(N=8)$, lack of blindness in Phase 3, and non-negligible dropout rate (7 out of 21 naïve) at the 1-year follow-up. Second, NIRS does not detect activity in deeper cortical structures, such as the anterior cingulate cortex, which is part of the neural network that subserves response inhibition (Duann et al, 2009). Third, as we expected, the NIRS signal amplitude decreased significantly on repeated measurement. We evaluated the NIRS signal of untreated HCs at the same time points as children with ADHD to control for the effects of repeated measurements. Because the crossover design was also applied in the comparison between MPH and placebo, any potential effects of learning on our results were considered to be resolved. Moreover, the primary aim of our study was the prediction of the effect of continuous administration of MPH based on the NIRS signal, and the use of repeated measurements was thus considered unlikely to affect our findings in this context. Although the clinical use of NIRS may be especially suitable in children because of its simplicity and safety, to date, no study has evaluated the reliability of repeated measurement of NIRS in children. It is necessary to further study the reliability of repeated NIRS measurement when using SST or other tasks in children. Finally, more study is required before this technique can be officially approved and applied in realworld clinical settings. The present findings should be replicated and validated in independent and larger subject groups in future multi-site studies. In particular, the differences among age, sex, and ADHD subtype should be evaluated in a large sample. It is also necessary to compare factors for predicting the effect of $\mathrm{MPH}$ combined with other medications such as atomoxetine in order to select the best treatment regimen for clinical application. Furthermore, a longitudinal study that follows the participants to adulthood, including those participants who stop treatment, is necessary to determine predictors for long-term success of treatments.

In conclusion, this study illustrates an innovative application of 'next-generation' neuroimaging to child psychiatry. Greater change in prefrontal activation after a single dose of $\mathrm{MPH}$ predicts greater effectiveness with continuous administration for $4-8$ weeks and 1 year. The present NIRS system may serve as a supplemental neuroimaging modality to aid in the clinical decision-making concerning continuous $\mathrm{MPH}$ administration in children with ADHD.

\section{FUNDING AND DISCLOSURE}

All authors have completed the ICMJE Form for Disclosure of Potential Conflicts of Interest. KK reports the following financial relationship. From 31 July 2003 to the present, the University of Tokyo and the Research and Developmental Centre, Hitachi Medical Corporation have had an official contract for a collaborative study on the clinical applications of near-infrared spectroscopy in psychiatric disorders, which has been approved by the Research Promotion Office,
University of Tokyo Hospital. For the present study, Hitachi Medical Corporation provided a project grant (JPY $300000 /$ year). SK is employed by Hitachi Medical Corporation and is also a contracted researcher at the University of Tokyo. AI-T, RT, YK, HK, and KK at the University of Tokyo and SK at Hitachi Medical Corporation developed the 'stimulus presentation device and stimulus task presentation method for optional measurement apparatus' (patent 2008-146721, Japan; patent 12996190). The University of Tokyo transferred this patent to Hitachi Medical Corporation. Hitachi Medical Corporation paid a transfer fee (JPY 100000 ) to the University of Tokyo. For the past 3 years, the authors declare the following Funding Statements. AI-T received a research grant from the Japan Medical Association. RT was a Newton International Fellow who was jointly funded by the Royal Society and the British Academy. YN was funded by the Takeda Science Foundation and MEXT. YK received research grants from the Mitsubishi Foundation, the Meiji-Yasuda Mental Health Foundation, and the Japan Society for the Promotion of Science. TI received research grants from the Ministry of Health, Labor and Welfare. KW was funded by a Grant-in-Aid for Scientific Research. HY was supported by Grants from the Japan Society for the Promotion of Science; CREST; the Adaptable and Seamless Technology Transfer Program; the Center of Innovation Program from the Japan Science and Technology Agency; and the Strategic Research Program for Brain Sciences from the Ministry of Education, Culture, Sports, Science, and Technology. NK was supported by the Japan Science and Technology Agency; CREST; Strategic Research Program for Brain Sciences by the Ministry of Education, Culture, Sports, Science and Technology of Japan; and the Japan Agency for Medical Research and Development. KK received research grants from Astellas, GSK, Dainippon-Sumitomo, Eisai, MSD, and Yoshitomi. YK received a research Grant for Comprehensive Research on Disability, Health, and Welfare; an Intramural Research Grant for Neurological and Psychiatric Disorders of NCNP; and a Grant-in-Aid for Scientific Research on Innovative Areas. The authors declare that over the past 3 years, the following payments were received. YN received compensation from the Tokyo Metropolitan Matsuzawa Hospital and AMED. YK has received honoraria for lectures at the Iwate Prefectural University, the Rissho University, the Japanese Society of Certified Clinical Psychologists, the Japanese Organization of Clinical Developmental Psychologists, the Education committee of Setagaya City, the Hizen Psychiatric Center, NHK Japan Broadcasting Corporation, and Nihon Bunka Kagakusha Corporation. $\mathrm{KH}$ has received compensation from the Matsubara Women's Clinic. HK has received compensation from the Department of Yokohama Rehabilitation Center, the Hongo-Todaimae kokorono Clinic, the Toshima Hospital, and the Metropolitan Bokutoh Hospital. TS has received compensation from the Fuchu Prison and the Kawasaki city recovery consultation office. AT has received compensation from the Hongo-todaimae mental clinic. TI has received compensation from MC Medical, TanabeMitsubishi, GSK, Taisyo-Toyama, Sanofi-Pasteur, Astellas, Chugai, Otsuka, Japan Vaccination, Meiji, Takeda, Kyorin, and MSD. KW has received honoraria for lectures from Eli Lilly, Phizer, and Janssen and has been given payment in 
exchange for clinical service in the Higashinagano Hospital, the Kiyose city Children developmental support center, the Koto city Educational Center, and the Tokyo University of Foreign Studies. In addition, KW has received a salary for delivering academic lectures at Ochanomizu University, the Japan College of Social Work, and Seikei University. HY has received compensation from Mitsubishi Cooperation and Teijin. NK has received honoraria for lectures from Meiji Seika, Eli Lilly, Yoshitomi, Pfizer, GSK, Astellas, Shionogi, and Mitsubishi Tanabe. KK has received honoraria for lectures by Daiichi-Sankyo, Otsuka, Meiji Seika, MSD, Astellas, Yoshitomi, Novartis, Eli Lilly, DainipponSumitomo, Janssen, GSK, and Pfizer and has received salary for clinical services at the Akasaka Clinic and The Clinic for Tokyo Securities Industry Health Insurance Society. YK has received honoraria for lectures and opinion hearing from Sumitomo Dainippon and/or for being a chairperson for Eli Lilly, Janssen, Astellas, and SHIONOGI. YK has received compensation as a supervisor from the Adachi Children Support Center 'Genki' and fees as adjunct professor at the Aizu Medical Center and Fukushima Medical University. The authors also declare that in the near future, AI-T anticipates receiving a grant from the Fulbright foundation and $\mathrm{KH}$ anticipates receiving compensation from Nayori City University. SO declared that except for income received from her primary employer, no financial support or compensation has been received from any individual or corporate entity over the past 3 years for research or professional service and that there are no personal financial holdings that could be perceived as constituting a potential conflict of interest. This study was supported by a grant from the Strategic Research Programme for Brain Sciences Project D: Development of biomarker candidates for social behavior and a Grant-in-Aid for Scientific Research on Innovative Areas (23118001 and 23118004; Adolescent Mind and SelfRegulation) from the MEXT, Japan to KK. This study was also supported in part by Health and Labor Sciences Research Grants, Comprehensive Research on Disability, Health and Welfare (H17-kokoro-Ippan-004 to NK, H20kokoro-Ippan-001 to KK, H20-kokoro-Ippan-003 to KK, and H23-seishin-Ippan-002 to RT, YN, and YK), Japan Society for the Promotion of Science, KAKENHI (Grant-in-Aid for Young Scientists (B) No. 24791201 and 26860913 to AI-T and No.23791309 \& 26860914 to RT), and Meiji-Yasuda Mental Health Foundation 2008 (Japan) to AI-T. This study was supported by the Brain/MINDS project of MEXT (to $\mathrm{KK}$ ).

\section{ACKNOWLEDGMENTS}

We thank all patients, healthy volunteers, and their families who participated in this study.

\section{Disclaimer}

The sponsors had no role in the design and conduct of the study; data collection, analysis, or interpretation; or in preparation, review, or approval of the manuscript. AI-T and KK had full access to all data in the study and take responsibility for the integrity of the data and the accuracy of the data analysis.

\section{REFERENCES}

Achenbach TM, Howell CT, Quay HC, Conners CK (1991). National survey of problems and competencies among four- to sixteen-year-olds: parents' reports for normative and clinical samples. Monogr Soc Res Child Dev 56: 1-131.

An L, Cao XH, Cao QJ, Sun L, Yang L, Zou QH et al (2013). Methylphenidate normalizes resting-state brain dysfunction in boys with attention deficit hyperactivity disorder. Neuropsychopharmacology 38: 1287-1295.

Borgwardt S, Fusar-Poli P (2012). Third-generation neuroimaging in early schizophrenia: translating research evidence into clinical utility. Br J Psychiatry 200: 270-272.

Buitelaar J, Medori R (2010). Treating attention-deficit/hyperactivity disorder beyond symptom control alone in children and adolescents: a review of the potential benefits of long-acting stimulants. Eur Child Adolesc Psychiatry 19: 325-340.

Buitelaar JK, Van der Gaag RJ, Swaab-Barneveld H, Kuiper M (1995). Prediction of clinical response to methylphenidate in children with attention-deficit hyperactivity disorder. J Am Acad Child Adolesc Psychiatry 34: 1025-1032.

Bush G, Spencer TJ, Holmes J, Shin LM, Valera EM, Seidman LJ et al (2008). Functional magnetic resonance imaging of methylphenidate and placebo in attention-deficit/hyperactivity disorder during the multi-source interference task. Arch Gen Psychiatry 65: 102-114.

Carter CS, Heckers S, Nichols T, Pine DS, Strother S (2008). Optimizing the design and analysis of clinical functional magnetic resonance imaging research studies. Biol Psychiatry 64: 842-849.

Cho SC, Hwang JW, Kim BN, Lee HY, Kim HW, Lee JS et al (2007). The relationship between regional cerebral blood flow and response to methylphenidate in children with attention-deficit hyperactivity disorder: comparison between non-responders to methylphenidate and responders. J Psychiatr Res 41: 459-465.

de Vries M, Geurts HM (2014). Beyond individual differences: are working memory and inhibition informative specifiers within ASD? J Neural Transm 121: 1183-1198.

Duann JR, Ide JS, Luo X, Li CS (2009). Functional connectivity delineates distinct roles of the inferior frontal cortex and presupplementary motor area in stop signal inhibition. J Neurosci 29: 10171-10179.

DuPaul GJ PT, Anastopoulos AD, Reid R. (1998). ADHD Rating Scale IV: Checklists, Norms, and Clinical Interpretation. Guilford: NY, USANY, USA.

Erder MH, Signorovitch JE, Setyawan J, Yang H, Parikh K, Betts KA et al (2012). Identifying patient subgroups who benefit most from a treatment: using administrative claims data to uncover treatment heterogeneity. J Med Econ 15: 1078-1087.

Frodl T, Skokauskas N (2012). Meta-analysis of structural MRI studies in children and adults with attention deficit hyperactivity disorder indicates treatment effects. Acta Psychiatr Scand 125: 114-126.

Gupta R, Kar BR (2009). Development of attentional processes in ADHD and normal children. Prog Brain Res 176: 259-276.

Guy W (1976). ECDEU Assessment Manual for Psychopharmacology -Revised. DHEW Publisher: BethesdaBethesdaVol ADM: 76-338.

Hannestad J, Gallezot JD, Planeta-Wilson B, Lin SF, Williams WA, van Dyck CH et al (2010). Clinically relevant doses of methylphenidate significantly occupy norepinephrine transporters in humans in vivo. Biol Psychiatry 68: 854-860.

Hart H, Radua J, Nakao T, Mataix-Cols D, Rubia K (2013). Metaanalysis of functional magnetic resonance imaging studies of inhibition and attention in attention-deficit/hyperactivity disorder: exploring task-specific, stimulant medication, and age effects. JAMA Psychiatry 70: 185-198.

Hermens DF, Cooper NJ, Kohn M, Clarke S, Gordon E (2005). Predicting stimulant medication response in ADHD: evidence 
from an integrated profile of neuropsychological, psychophysiological and clinical factors. J Integr Neurosci 4: 107-121.

Hermens DF, Rowe DL, Gordon E, Williams LM (2006). Integrative neuroscience approach to predict ADHD stimulant response. Expert Rev Neurother 6: 753-763.

Ilgin N, Senol S, Gucuyener K, Gokcora N, Sener S (2001). Is increased D2 receptor availability associated with response to stimulant medication in ADHD. Dev Med Child Neurol 43: $755-760$.

Ishii-Takahashi A, Takizawa R, Nishimura Y, Kawakubo Y, Kuwabara H, Matsubayashi J et al (2013). Prefrontal activation during inhibitory control measured by near-infrared spectroscopy for differentiating between autism spectrum disorders and attention deficit hyperactivity disorder in adults. Neuroimage Clin 4: 53-63.

Konrad K, Neufang S, Fink GR, Herpertz-Dahlmann B (2007). Long-term effects of methylphenidate on neural networks associated with executive attention in children with ADHD: results from a longitudinal functional MRI study. J Am Acad Child Adolesc Psychiatry 46: 1633-1641.

Krause J (2008). SPECT and PET of the dopamine transporter in attention-deficit/hyperactivity disorder. Expert Rev Neurother 8: 611-625.

la Fougere C, Krause J, Krause KH, Josef Gildehaus F, Hacker M, Koch W et al (2006). Value of 99mTc-TRODAT-1 SPECT to predict clinical response to methylphenidate treatment in adults with attention deficit hyperactivity disorder. Nucl Med Commun 27: 733-737.

Lee SH, Song DH, Kim BN, Joung YS, Ha EH, Cheon KA et al (2009). Variability of response time as a predictor of methylphenidate treatment response in korean children with attention deficit hyperactivity disorder. Yonsei Med J 50: 650-655.

Monden Y, Dan H, Nagashima M, Dan I, Kyutoku Y, Okamoto M et al (2012). Clinically-oriented monitoring of acute effects of methylphenidate on cerebral hemodynamics in ADHD children using fNIRS. Clin Neurophysiol 123: 1147-1157.

Otsubo T, Tanaka K, Koda R, Shinoda J, Sano N, Tanaka S et al (2005). Reliability and validity of Japanese version of the MiniInternational Neuropsychiatric Interview. Psychiatry Clin Neurosci 59: 517-526.

Ponseti J, Granert O, Jansen O, Wolff S, Beier K, Neutze J et al (2012). Assessment of pedophilia using hemodynamic brain response to sexual stimuli. Arch Gen Psychiatry 69: 187-194.

Rapport MD, Denney C, DuPaul GJ, Gardner MJ (1994). Attention deficit disorder and methylphenidate: normalization rates, clinical effectiveness, and response prediction in 76 children. J Am Acad Child Adolesc Psychiatry 33: 882-893.
Rubia K, Alegria AA, Cubillo AI, Smith AB, Brammer MJ, Radua J (2014). Effects of stimulants on brain function in attentiondeficit/hyperactivity disorder: a systematic review and metaanalysis. Biol Psychiatry 76: 616-628.

Rubia K, Halari R, Mohammad AM, Taylor E, Brammer M (2011). Methylphenidate normalizes frontocingulate underactivation during error processing in attention-deficit/hyperactivity disorder. Biol Psychiatry 70: 255-262.

Sangal RB, Sangal JM (2004). Attention-deficit/hyperactivity disorder: cognitive evoked potential (P300) topography predicts treatment response to methylphenidate. Clin Neurophysiol 115: 188-193.

Sawada M, Iida J, Ota T, Negoro H, Tanaka S, Sadamatsu M et al (2010). Effects of osmotic-release methylphenidate in attentiondeficit/hyperactivity disorder as measured by event-related potentials. Psychiatry Clin Neurosci 64: 491-498.

Schlochtermeier L, Stoy M, Schlagenhauf F, Wrase J, Park SQ, Friedel E et al (2011). Childhood methylphenidate treatment of ADHD and response to affective stimuli. Eur Neuropsychopharmacol 21: 646-654.

Schweitzer JB, Lee DO, Hanford RB, Tagamets MA, Hoffman JM, Grafton ST et al (2003). A positron emission tomography study of methylphenidate in adults with ADHD: alterations in resting blood flow and predicting treatment response. Neuropsychopharmacology 28: 967-973.

Shaw P, Sharp WS, Morrison M, Eckstrand K, Greenstein DK, Clasen LS et al (2009). Psychostimulant treatment and the developing cortex in attention deficit hyperactivity disorder. Am J Psychiatry 166: 58-63.

Stoy M, Schlagenhauf F, Schlochtermeier L, Wrase J, Knutson B, Lehmkuhl U et al (2011). Reward processing in male adults with childhood ADHD-a comparison between drug-naive and methylphenidate-treated subjects. Psychopharmacology (Berl) 215: 467-481.

Takizawa R, Kasai K, Kawakubo Y, Marumo K, Kawasaki S, Yamasue $\mathrm{H}$ et al (2008). Reduced frontopolar activation during verbal fluency task in schizophrenia: a multi-channel nearinfrared spectroscopy study. Schizophr Res 99: 250-262.

Volkow ND, Fowler JS, Wang G, Ding Y, Gatley SJ (2002). Mechanism of action of methylphenidate: insights from PET imaging studies. J Atten Disord 6(Suppl 1): S31-S43.

Wilens TE, Adler LA, Adams J, Sgambati S, Rotrosen J, Sawtelle R et al (2008). Misuse and diversion of stimulants prescribed for ADHD: a systematic review of the literature. J Am Acad Child Adolesc Psychiatry 47: 21-31.

Zeiner P, Bryhn G, Bjercke C, Truyen K, Strand G (1999). Response to methylphenidate in boys with attention-deficit hyperactivity disorder. Acta Paediatr 88: 298-303.

Supplementary Information accompanies the paper on the Neuropsychopharmacology website (http://www.nature.com/npp) 